\title{
VERHALTENSKODIZES FUR MULTINATIONALE KONZERNE UND DIE INTERESSENPOSITION DER ENTWICKLUNGSLÄNDER
}

\author{
Von Alphons Studier
}

\section{Einleitung}

Die hauptsächlich in den Vereinten Nationen geführte Diskussion über die neue Weltwirtschaftsordnung, in deren Kontext auch die Auseinandersetzung zwischen Industrie- und Entwicklungsländern über Verhaltenskodizes für multinationale Konzerne (MNK) steht, stellt die bisher umfassend'ste Infragestellung bestehender politisch-ökonomischer Strukturen des internationalen Systems und tradierter völkerrechtlicher Prinzipien durch die Dritte Welt dar. Zwar bot sich den Entwicklungsländern in der Organisation der Vereinten Nationen die Möglichkeit kollektiver Interessenartikulierung vor einem weltöffentlichen und universellen Forum; die ursprünglich nur auf die Erfordernisse eines begrenzten Kreises ,,christlicher" bzw. ,,zivilisierter" Nationen zugeschnittenen traditionellen Völkerrechtsregeln hatten jedoch weiterhin Bestand ${ }^{1}$. Die aus dieser Situation resultierenden Konflikte lassen sich letztlich aber erst vor dem Hintergrund divergierender ökonomischer Interessen zwischen Industrie- und Entwicklungsländern verstehen, der zugleich die entscheidende Bestimmungsgröße der Formulierung von Verhaltenskodizes bildet. Die Anerkennung der Entwicklungsländer als besondere Völkerrechtssubjekte in der Resolutionspraxis der UN ${ }^{2}$ stellt insofern keine Privilegierung dieser Länder dar, sondern reflektiert lediglich ihre ökonomischen Besonderheiten.

Die Besonderheiten unterentwickelter Länder werden von der neueren Forschung weniger in typischen Zustandsindikatoren als in gemeinsamen genetisch strukturellen Merkmalen des abhängigen Kapitalismus gesehen ${ }^{3}$. Diese Faktoren sind einmal die strukturelle Heterogenität und zum anderen ein deformiertes Reproduktionsschema, die beide sowohl auf historische Gründe der Unterentwicklung als auch gegenwärtige Entwicklungshindernisse verweisen. Während die strukturelle Heterogenität die unorganische Úberlagerung verschiedener (historischer) Produktionsweisen bezeichnet, erfaßt der Begriff deformiertes Reproduktionsschema die entwicklungsländerspezifische Verbindung von Luxusgüter- und exportorientierter Produktion. Kennzeichnend für den abhängigen Kapitalismus ist folglich eine auf die Bedarfsdeckung der Industrieländer ausgerichtete, inhomogene Produktionsstruktur, die nur geringe gesamtgesellschaftliche Entwicklungsimpulse hervorbringt. Folgt man dem größten Teil der vorliegenden Literatur ${ }^{4}$, so führt die MNK-Tätigkeit in der Dritten Welt durchweg zu einer weiteren Verschlechterung der skizzierten Situation. Gerade die naturwüchsige Penetration der Entwicklungsländer-Ökonomien durch MNK, die meist auf

\footnotetext{
1 Vgl. hierzu u. a. O. Udokang, The Role of the New States in International Law, in: Archiv des Völkerrechts 1971/72, S. 145 ff.; W. D. Verwey, Economic Development, Peace and International Law, Assen 1972, S. $236 \mathrm{ff}$.

2 Vgl. Verwey, Economic Development ..., a.a.O., S. 265 ff.

3 Siehe hierzu und zum folgenden für alle: D. Senghaas, Weltwirtschaftsordnung und Entwicklungspolitik, Frankfurt/M. 1977

$4 \mathrm{Vgl}$. ausführlich zu den empirischen Befunden und zur eingehenden Diskussion der vielfältigen Probleme, die an diesem Ort nicht aufgezeigt werden können, aus der zahllosen MNK-Literatur insbesondere: D. Senghaas/U. Menzel (Hrsg.), Multinationale Konzerne und Dritte Welt, Opladen 1976; V. Bethke/G. Koopmann, Multinationale Unternehmen und Entwicklungsländer, Hamburg 1975 ; R. Müller, Poverty is the Product, in: Foreign Policy, No. 13 (Winter 1973-74), S. 71 ff.; United Nations, Department of Economic and Social Affairs, Multinational Corporations in World Development, New York 1973 (ST/ECA/190); United Nations, Centre on Transnational Corporations, Transnational Corporations in World Development: A Re-examination, New York 1978 (E/C. 10/38)
} 
einer hochakkumulierten Kapitalbasis und mit high sophisticated technology operieren, führt in den meisten Fällen nolens volens ${ }^{5}$ dazu, daß die Entfaltungsmöglichkeiten der ohnehin nur rudimentären einheimischen Bourgeoisie noch weiter schrumpfen. Die einheimischen Unternehmen entfallen als Entwicklungsträger, wenn es MNK gelingt, die vorhandenen natürlichen, menschlichen und finanziellen Ressourcen weitgehend zu monopolisieren und ungestört auszubeuten. Dabei führt der durch MNK initiierte offene und versteckte (Transferpreise, Lizenzgebühren u. dgl.) Ressourcentransfer zur weiteren Schwächung nationaler Volkswirtschaften. Uberdies verhindern oft restriktive Geschäftspraktiken von MNK und deren patentrechtliche Produktionsmonopole Ansätze zu einer selbstinduzierten Entwicklung. Von wesentlicher Bedeutung für die Überwindung der Unterentwicklung ist es insofern, ob es gelingt, MNK in eine gesamtgesellschaftlich verpflichtete Rahmenplanung peripherer Staaten steuernd einzubinden.

Um diese Planung auch gegebenenfalls gegen das Interesse und den erklärten Willen von MNK durchsetzen zu können, wird die Stärkung der Verhandlungsmacht der Entwicklungsländer gegenüber MNK von wesentlicher Bedeutung sein. Zur Verbesserung ihrer bargaining power nun könnten Verhaltenskodizes für MNK unter bestimmten Voraussetzungen einen Beitrag leisten. Gegenstand der folgenden Untersuchung wird daher sein zu ermitteln, inwieweit die bislang vorgeschlagenen internationalen Verhaltenskodizes für MNK in dem genannten Sinn als Mittel zum Zweck der Entwicklung fungieren können.

Neben diesem allgemeinen Gradmesser wird als konkretes Unterscheidungskriterium im folgenden zusätzlich auf einen Vorschlag des Centre on Transnational Corporations rekurriert. Das Centre hat zur Unterscheidung der Effektivität verschiedener Formulierungsmöglichkeiten des UN-Kodex für MNK, der gegenwärtig von der Commission on Transnational Corporations diskutiert wird ${ }^{6}$, drei Gestaltungsebenen genannt ${ }^{7}$, die auch anwendbar sind zur Charakterisierung bisheriger Regulierungsversuche für MNK. Danach lassen sich als Formulierungsmöglichkeiten folgende Lösungen denken: erstens entweder eine rechtlich verbindliche Konvention oder nur unverbindliche Empfehlungen; zweitens entweder konkrete Bezeichnung des wünschenswerten Verhaltens bzw. der Verbote oder allgemein gehaltene Umschreibungen; drittens entweder Uberprüfung des Konzernverhaltens vor einem internationalen Forum oder Verweis auf die jeweils in Frage kommende nationale Gerichtsbarkeit. Die maximale Gestaltungsmöglichkeit läuft auf eine bindende, konkret formulierte und international durchgesetzte Konvention hinaus, das Minimum bestünde in allgemein formulierten Empfehlungen, die von den einzelnen Staaten dann noch gesondert national kodifiziert werden müßten. Kombinationen einzelner Elemente sind natürlich denkbar. Es dürfte ohne nähere Erläuterung einsichtig sein, daß der maximale approach im allgemeinen in dem Interesse der Entwicklungsländer und der minimale im MNK-Interesse liegt. Insofern bietet sich diese Unterscheidung als weiteres Einordnungskriterium an.

Anhand der beiden genannten Fragestellungen sollen zunächst internationale Verhaltenskodizes für MNK, die im Mittelpunkt dieser Untersuchung stehen, untersucht werden. Zunächst wird auf die Havanna-Charta sowie verschiedene von privater Seite und Organisationen der Industrieländer vorgebrachte Kodizes eingegangen. Daran schließt sich eine Darstellung der im UN-Rahmen geführten Diskussion, die gegenwärtig zentral in der Commis-

\footnotetext{
5 Zum volens siehe auch als anschauliche Darstellung eines brasilianischen mittelständischen Unternehmers: K. R. Mirow, Die Diktatur der Kartelle, Reinbek b. Hamburg 1978

$6 \mathrm{Zu}$ Einzelheiten hierzu siehe Abschnitt 2.3.2

7 Siehe United Nations, Centre on Transnational Corporations, Transnational Corporations: Issues Involved in the Formulation of a Code of Conduct, New York 1976, S. 16 f. und 35 ff. (E/C. 10/17), (bei Zitatwiederholungen von UN-Veröffentlichungen wird im folgenden jeweils das betreffende UN-Symbol angeführt); vgl. auch G. Berweger, Verhaltensrichtlinien für multinationale Konzerne, in: ,NNeue Zürcher Zeitung“, FA (NZZ) v. 17. 8. und 19. 8. 77
} 
sion on Transnational Corporations ausgetragen wird, an. Die Möglichkeiten und Grenzen regionaler bzw. nationaler Investitionsgesetzgebung sollen anhand des Andenpakts und Vietnams aufgezeigt werden. Abschließend sollen einige theoretische Verallgemeinerungen vorgenommen werden.

\section{Internationale Kodizes für MNK}

\subsection{Die Havanna-Charta von 1948}

Nachdem bereits im Völkerbund erste Versuche unternommen worden waren, das Problem international operierender Konzerne aufzugreifen, wurde dieses Problem im Rahmen des UN-Systems zuerst in der Havanna-Charta 1948 behandelt. Bekanntlich scheiterte die Havanna-Charta, die zur Schaffung einer eigenständigen Handelsorganisation (ITO) ausgehandelt wurde, an der Weigerung der USA, die Charta zu ratifizieren. Von diesen Bemühungen blieb als fragmentarisches Provisoriumlediglich das GATT übrig. In den Art. 12 (internationale Investitionen für ökonomische Entwicklung und Wiederaufbau), 46-54 (restriktive Geschäftspraktiken) und 72 (Informationssystem) der Havanna-Charta sind bereits Bestimmungen enthalten, die ansatzweise einen Kodex für Auslandskapital beinhalten ${ }^{8}$. Zunächst wird in Art. 12 grundsätzlich festgestellt, daß Auslandsinvestitionen von großem Wert für die Förderung wirtschaftlicher Entwicklung seien. Eingriffe gegenüber widrigen Auswirkungen dieser Investitionen werden den Staaten in allgemeiner Form freigestellt. So haben die Mitgliedstaaten das Recht ,,i) to take any appropriate safeguards necessary to ensure that foreign investment is not used as a basis for interference in its internal affairs or national policies; ii) to determine whether and to what extent and upon what terms it will allow future foreign investment; iii) to prescribe and give effect on just terms to requirements as to the ownership of existing and future investments $(\ldots)^{\text {" } 9}$. Andererseits sollen die Staaten den Investoren aber auch einen weitreichenden Investitionsschutz gewähren. Gastländer sollen sich dafür verpflichten, ,,i) to provide reasonable opportunities for investments acceptable to them and adequate security for existing and future investments, and ii) to give due regard to the desirability of avoiding discrimination as between foreign investments 10"'. Art. 72 nennt unter den Funktionen der ITO u. a., ,, a) to collect, analyse and publish information relating to international trade, including information relating to commercial policy, business practices, commodity problems and industrial and general economic development; (...) c) to undertake studies, and (...) measures designed i) to assure just and equitable treatment for foreign nationals and enterprises".

Sicherlich ist es in einem ideengeschichtlichen Sinn richtig, die Elemente der HavannaCharta in bezug auf MNK als Ausgangspunkt der heutigen Diskussion über Verhaltenskodizes zu sehen ${ }^{11}$. Allerdings gehen Forderungen, die darauf hinauslaufen, die HavannaCharta als organisatorisches Substrat zur Aufrechterhaltung einer liberalen Weltwirtschaftsordnung wiederzubeleben ${ }^{12}$, an den gegenwärtigen Realitäten vorbei. Da Entwick-

8 Die Havanna-Charta ist abgedruckt in: United Nations Conference on Trade and Employment held at Havana Cuba from November 21, 1947, to March 24, 1948. Final Act anf Related Documents, Havana 1948 (E/Conf. 2/78).

9 Ebd., Art. 12.

10 Ebd.

11 Vgl. The Impact of Multinational Corporations on the Development Process and on International Relations, Report of the Group of Eminent Persons to Study the Role of Multinational Corporations on Development and on International Relations, 24 May 1974 (E/5500/Add. 1), abgedruckt in: International Legal Materials (ILM) 1974, S. 800 ff., hier: S. 833; vgl. auch E. Piehl, Stichwort Multinationale Konzerne, in: R. Wolfrum u. a. (Hrsg.), Handbuch Vereinte Nationen, München 1977, S. 298.

12 So etwa K. Biedenkopf, Auf dem Weg zu einer freien Weltwirtschaftsordnung, in: Multinationale im Nord-Süd-Konflikt, Frankfurt/M. 1976, S. 29 und passim. 
lungsländer-Forderungen bei der Formulierung der Havanna-Charta kaum berücksichtigt wurden und diese Charta dem für die ersteren essentiellen Prinzip nationaler Souveränität nicht genügend Geltung verschafft, wird die Havanna-Charta von diesen Ländern als nicht mehr akzeptabel betrachtet. Insofern steht die Havanna-Charta heute nicht mehr zur Diskussion $^{13}$.

\subsection{Von privater Seite und von Industrieländern formulierte Kodizes}

Nach der gescheiterten Havanna-Charta wurde in den Vereinten Nationen die Diskussion über allgemeine Richtlinien für MNK mehr als zwei Jahrzehnte unterbrochen. Zwischenzeitlich wurden lediglich Teilbereiche, so u. a. 1953 im Wirtschafts- und Sozialrat die Frage restriktiver Geschäftspraktiken und 1952 bzw. 1962 in der Vollversammlung die ständige Souveränität über natürliche Ressourcen ${ }^{14}$, behandelt. Erst die MNK-Expansion in den 60er Jahren ließ die Gesamtproblematik signifikant werden. Kritik an MNK wurde sowohl in Industrieländern, hier insbesondere von den Gewerkschaften, als auch in den Entwicklungsländern, hier vor allem durch die lateinamerikanischen Dependenztheoretiker - deren Erkenntnisse sich u. E. auch in den MNK-Statuten des Andenpakts niederschlugen -, vorgetragen. Erste umfassende Regulierungskonzeptionen für MNK, wie etwa die Schaffung eines für Industrieländer geltenden ,,GATT for Investment" ${ }^{\text {"15, }}$, wurden vorgeschlagen. Diese Konzeptionen wurden zu Beginn der 70er Jahre auch von den MNK selbst aufgegriffen, die damit eine bemerkenswerte konzeptionelle Änderung vornahmen. Wurde zuvor - d. h. von den 30er bis in die 60er Jahre - von privater Seite an Konventionsentwürfen gearbeitet, die primär auf den Schutz von ausländischen Privatinvestitionen zielten ${ }^{16}$, so wurde nun auf dem Weg einer freiwilligen Selbstbeschränkung versucht, über die Ausarbeitung allgemeiner Verhaltenskodizes der öffentlichen Kritik den Wind aus den Segeln zu nehmen. Das vorrangige Ziel des Investitionsschutzes sollte damit allerdings nur auf indirektem Weg weiterhin verfolgt werden.

\subsubsection{Der Kodex des Pacific Basin Economic Council}

Illustrierend für diese Tendenz ist die 1972 vom Pacific Basin Economic Council (PBEC) vorgelegte „Pacific Basin Charter on International Investments“"17. Die Charta des PBEC, dessen Mitglieder aus international operierenden Unternehmen Australiens, Kanadas, Japans, Neuseelands und der Vereinigten Staaten bestehen, umfaßt insgesamt 36 Artikel. In der Präambel werden als Adressaten einerseits Regierungen, andererseits MNK genannt. Als Zielsetzung wird sowohl die Begünstigung raschen und sinnvollen ökonomischen Wachstums als auch die Stärkung des freien Unternehmertums angeführt. Der Anschein einer ausgewogenen Grundsatzerklärung, den sich die PBEC-Charta auch in den einzelnen Artikeln zu geben versucht, erweist sich allerdings als trügerisch. So wenden sich 21 Artikel haupt-

13 In diesem Sinn auch F. Schlupp, Konzeptionen und Konsequenzen administrativer Maßnahmen zur Uberwachung und Kontrolle Multinationaler Konzerne, in: D. Senghass/V. Menzel (Hrsg.), Multinationale Konzerne . . ., a. a. O., S. 201.

14 UNGA-Res. 626 (VII) und 1803 (XVII).

15 A. Goldberg/C. P. Kindleberger, Toward a GATT for Investment: A Proposal for Supervision of the International Corporation, in: Law and Policy in International Business 1970, S. $295 \mathrm{ff}$.

16 Vgl. die Aufzählung bei G. Langer, Können multinationale Unternehmen international kontrolliert werden?, in: D. Kebschull/O. G. Mayer (Hrsg.), Multinationale Unternehmen. Anfang oder Ende der Weltwirtschaft?, Frankfurt/M. 1974, S. 234, Anm. 53. Vgl. auch G. Schwarzenberger, Der Schutz von Auslandsinvestitionen, Bad Homburg - Berlin - Zürich 1969.

17 Text in "Fortune“" v. September 1972, S. 52 f. Auszugsweise abgedruckt in: „Blick durch die Wirtschaft“ v. 11. 9. 72. 
sächlich an die Regierungen der Heimat- und vor allem der Gastländer. Priorität hat dabei die Forderung einer fast vollständigen Inländerbehandlung von MNK, also einem Diskriminierungsverbot gegenüber inländischen Unternehmen, das sich u. a. von Besteuerung über Zugang zu Kapitalmärkten bis zum Kapitaltransfer erstrecken soll. Die rigide Einhaltung dieses Prinzips würde die Planungskapazität der Gastländer und damit auch deren Verhandlungsposition gegenüber MNK schwächen. Zudem sind die Grundsätze, die sich an MNK wenden, etwa die Mitwirkung von MNK an der Erfüllung nationaler Entwicklungspläne (Art. A.1.), die Achtung nationaler souveräner Rechte (Art. B.1.) oder die Einhaltung nationalen Rechts (Art. C.1.) - Prinzipien, die weitgehend unbestritten sind -, nur allgemein formuliert. Die Úberprüfung der korrekten Anwendung und Beachtung der Pacific Basin Charter soll bei Streitfällen in bilateralen Konsultationen zwischen MNK und Gastländern geklärt werden, und - sofern keine Einigkeit erzielt werden kann - einer dritten Partei vorgelegt werden, wobei ausdrücklich auf das International Center for the Settlement of Investment Disputes verwiesen wird (Art. C.8.1.) ${ }^{\mathbf{1 8}}$. Da ein nach den prozeduralen Regeln dieses Weltbank-Centers ausgetragener Investitionsstreit mit einem partiellen Souveränitätsverlust der Entwicklungsländer verbunden ist, wird diese Lösung aus ihrer Sicht sowenig befriedigen können wie die PBEC-Charta insgesamt.

\subsubsection{Die Guidelines der Internationalen Handelskammer}

Konzeptionell sehr ähnlich, inhaltlich aber ausführlicher, sind die 1972 von der Internationalen Handelskammer (ICC) angenommenen „Leitsätze für Auslandsinvestitionen“, die sich seit 1970 in der Diskussion befanden ${ }^{19}$. Wie die PBEC-Charta, wenden sich auch die ICC-Guidelines sowohl an MNK als auch an die Heimat- und Gaststaaten als Adressaten; während die ICC aber an die Heitmat- und insbesondere Gastländer appelliert, sich weitgehend interventionistischer Politik zu enthalten, werden MNK nur in allgemeiner und unverbindlicher Form zu ökonomischer Kooperation aufgefordert. So wird als Ziel die ,,Förderung eines Klimas gegenseitigen Vertrauens" formuliert ${ }^{20}$. Von überragender Bedeutung soll dabei auf seiten der Gastländer die Gewährleistung nichtdiskriminierender Behandlung von MNK sein, worauf in zahllosen Artikeln hingewiesen wird. Zum anderen sollen die Prinzipien der freien Marktwirtschaft gefestigt werden. In Abschnitt III. 3. a) und b) wird postuliert, daß die Regierung des Gastlandes weitgehend ,,keine Beschränkung für die Überweisung von Darlehenszinsen, Rückzahlungen, Dienstleistungs- und Beratungsgebühren, Lizenzgebühren und ähnlichen Zahlungen auferlegen“ und ,,dem Investor den freien Gewinntransfergestatten" sollte. Der Abfluß finanzieller Ressourcen würde demnach völlig ungehindert stattfinden können. Ferner soll MNK der Import von wesentlichen Bedarfsgütern ,,ohne unnötige Formalitäten und ohne übermäßige Zoll- und sonstige Abgaben“" gestattet werden ${ }^{21}$. Die Realisierung dieser Bestimmung würde an die Wurzel der importsubstituierenden Industrialisierungspolitik gehen, mit der Entwicklungsländer eigenständiges ökonomisches Wachstum zu stimulieren versuchen. Schließlich sollen ,, neue Investitionen nicht entmutigt werden“, ,,zwangsweise erfolgende staatliche Beteiligung am Kapital oder an der Geschäftsführung von (multinationalen) Unternehmen (nicht versucht

18 Vgl. hierzu: G. Langer, Das Weltbank-Ubereinkommen zur Beilegung von Investitionsstreitigkeiten, in: Außenwirtschaftsdienst (AWD) 1972 , S. $321 \mathrm{ff}$.

19 International Chamber of Commerce, Guidelines for International Investment, o. O. (Paris) 1972 (ICC-Brochure 272). Deutscher Text in: Außenwirtschaft 1973, S. 396 ff.

20 Ebd., Einleitung.

21 Ebd., Abschnitt VIII.3.C). 
werden) zu erreichen“, und versucht werden ,,Mißbrauch einer beherrschenden Marktstellung (...) durch die Förderung des Wettbewerbs, vor allem durch die Anregung neuer Investitionen, zu begegnen oder durch die Senkung der Einfuhrzölle22“". Das hieße den Teufel mit dem Beelzebub auszutreiben. Z. T. richten sich diese letztgenannten Bestimmungen explizit gegen die entsprechenden Vorschriften des Andenpakts. Den ICC-Guidelines soll insofern eine Modellfunktion zukommen, wie es auch in deren Einleitung heißt, um insbesondere die Diskussion in den Vereinten Nationen zu beeinflussen. Zusätzlich werden die Regierungen aufgefordert, zum Zweck des Eigentumsschutzes ,, auf bilateraler oder multilateraler Grundlage verbindliche völkerrechtliche Vereinbarungen (. . .) einzugehen ${ }^{23}$ ". Damit sollen offenbar faits accomplis für diese Diskussion über die MNK geschaffen werden ${ }^{24}$. In bezug auf die MNK selbst zeigen die ICC-Guidelines ebenfalls einen nahezu ridikülen Ultraliberalismus. Es werden zwar inzwischen allgemein anerkannte und unbestrittene Forderungen, wie etwa die befriedigende Einfügung von Investitionen in nationale Entwicklungspläne (Abschn. I.1.a), das Eingehen von joint ventures (II. 1.a), oder die Beachtung inländischer Gesetze (V. 1.a), erhoben. Andererseits aber bleibt der Umfang veröffentlichter Informationen den MNK anheimgestellt (I.1.d); bei Transfers finanzieller Mittel kann die Zahlungsbilanzposition des Gastlandes praktisch unberücksichtigt gelassen bleiben (III.1.a); die Höhe der Reinvestionsquote bleibt ebenfalls MNK überlassen (III. 1.d); partiell werden sogar restriktive Geschäftspraktiken legitimiert (VIII.1.b); und schließlich ergeht an MNK der bloße Appell, das Transferpreissystem durch eine ,,faire Preispolitik“ zu ersetzen (VIII. 1.c). Derartige schwache Regelungen werden überdies noch auf eine fakultative Basis gestellt. Es dürfte deutlich geworden sein, daß ein solcher Kodex den Interessen der Entwicklungsländer diametral zuwiderläuft.

\subsubsection{Die OECD-Richtlinien für MNK}

Abgesehen von weiteren, ähnlich konzipierten Vorschlägen privater Seite - wie etwa dem Entwurf der US-Chamber of Commerce von $1975^{25}$ oder Papieren, die von einzelnen MNK vorgelegt worden sind ${ }^{\mathbf{2 6}}$, wurde die Diskussion über Verhaltenskodizes auch in Industrieländern verstärkt geführt. So wurden Entwürfe für Kodizes u. a. im Rahmen der EG und zwischen dem Europa-Parlament und dem US-Kongreß beraten ${ }^{27}$. In diesen Kontext ordnen sich auch die OECD-Richtlinien von 1976 ein $^{28}{ }^{29}$. Schon die äußere Form der OECD-Declaration weist auf das gängige Verfahren des package dealings hin. Zunächst richtet sie sich im ersten Teil sowohl an MNK, denen die Einhaltung der Guidelines empfohlen wird, als auch an die Heimat- und Gastländer, die bestimmte Verfahren in bezug auf Di-

\footnotetext{
22 Abschnitte II.3.d), e) und f).

23 Abschnitte I.2.b) und I.3.f).

24 Gerade an dem Streit über die Anwendung tradierter enteignungsvölkerrechtlicher Regeln nach dem internationalen Mindeststandard des Fremden einerseits und dem neuen völkerrechtlichen Prinzip ständiger Souveränität über natürliche Ressourcen andererseits, wird die Herausforderung überkommenen Völkerrechts durch die Entwicklungsländer überaus deutlich.

25 United States Chamber of Commerce, Elements of Global Business Conduct for Possible Inclusion in Individual Company Statements, Washington 1975.

26 Bsplw.: Caterpillar Tractor Company, A Code of World Business Conduct, Peoria (Ile.) 1975.

27 Vgl. U. Holtz, Europa und die Multis, Baden-Baden 1978, S. 85 ff.; NZZ v. 21. 9. 77.

28 Declaration on International Investment and Multinational Enterprises, OECD Press Release A (76) 20 v. 21. 6. 76, abgedruckt in: ILM 1976, S. $967 \mathrm{ff}$. Die Guidelines for Multinational Enterprises sind aufS. $969 \mathrm{ff}$. abgedruckt. Deutscher Text beiB. Grossfeld/U. Hübner, Erklärung und Leitsätze der OECD für multinationale Unternehmen, in: Zeitschrift für Unternehmens- und Gesellschaftsrecht $1 / 78$, S. $156 \mathrm{ff}$.

29 Vgl. zu den OECD-Guidelines allgemein: M. Holthus, Pflichtübungen der OECD in Sachen Multis, in: Wirtschaftsdienst 1976, S. 324 f.; H. Schwamm/D. Germidis, Les codes de conduite pour les entreprises multinationales: qu' en est-il?, Brüssel 1977; B. Grossfeld/U. Hübner, Erklärung . . . a. a. O., S. 164 ff.
} 
rektinvestitionen gewährleisten sollen. Die sich daran anschließenden auf freiwilliger und rechtlich unverbindlicher Basis beruhenden Guidelines wenden sich dann zur Befolgung allgemeiner Grundsätze sowie Vorschriften zu Informationsdarlegung, Wettbewerb, Finanzierung, Besteuerung, Beschäftigung und Technologietransfer sowohl hauptsächlich an $\mathrm{MNK}$ als auch an inländische Unternehmen. Im Gegensatz zu diesen Richtlinien sind die abschließenden drei Ratsbeschlüsse der OECD zu Konsultationsverfahren zwischen den Mitgliedstaaten, der Inländerbehandlung und Maßnahmen zur Förderung oder Verhinderung von Direktinvestitionen ${ }^{30}$ rechtswirksam. Schon an dieser Konstruktion wird sichtbar, daß hier mit unterschiedlicher Elle gemessen wurde. Darüber hinaus enthält die Declaration aber auch einen inhaltlichen Widerspruch, wenn sie auf der einen Seite die rigide Einhaltung des Nichtdiskriminierungsverbots von MNK qua Inländerbehandlungsgebot postuliert, andererseits aber schon durch die bloße Existenz der Richtlinien belegt, daß MNK ein Spezialproblem bilden, das ggf. auch diskriminierende staatliche Maßnahmen erfordert.

Nicht minder vage und zweifelhaft sind auch die meisten anderen Bestimmungen ausgefallen. So sind die allgemeinen Prinzipien bezüglich der Beachtung nationaler wirtschaftspolitischer Ziele durch MNK durchweg äußerst allgemein formuliert. Ferner reflektiert der Wettbewerbsabschnitt nur die ohnehin in Industrieländern vorhandenen gesetzlichen Bestimmungen zur Verhinderung von Wettbewerbsbeschränkungen. Absolut unzureichend ist die Bestimmung zur MNK-Finanzierung, wonach MNK die Zahlungsbilanzsituation und Kreditpolitik des Gastlandes lediglich in Erwägung ziehen sollen. Fraglich ist es weiterhin, wenn es heißt, MNK sollten Belegschaftsvertreter über die Unternehmenspolitik nur informieren, soweit dies im Rahmen vorhandener Gesetze und Praktiken bleibt, und ,,observe standards of employment and industrial relations not less favourable than those observed by comparable employers in the host country". Soll dies die Anpassung an in autoritären Staaten üblichen Umgangsformen legitimieren? Sehr schwach ausgefallen ist ferner die Ratsentscheidung über Konsultationsverfahren. So kann auf Vorschlag eines Mitgliedstaates einem MNK (!), unter der Voraussetzung, daß dieser dies wünscht (!), die Gelegenheit gegeben werden, sich vor einem speziellen OECD-Komitee zur Anwendung der Guidelines zu äußern. Bei soviel Konzilianz ist es nicht weiter überraschend, daß sich die meisten MNK die OECD-Declaration zu eigen gemacht haben. Dem stehen auch nicht die relativ weitreichenden Bestimmungen bezüglich Bestechungspraktiken und Informationsoffenlegung entgegen. Vor dem Hintergrund der Lockheed-Bestechungsaffären sind die Formulierungen zu den Bestechungsverboten auf Betreiben der USA sogar noch wesentlich gegenüber den Entwürfen zum OECD-Kodex verschärft worden ${ }^{31}$. War in den Entwürfen noch die sehr doppeldeutige Formulierung enthalten, die MNK sollten ,,within the framework of laws and regulations of the host country, observe the best standards set by relevant local customs and practices with regard to rendering gifts and other benefits to public servants", so heißt es in den endgültigen Guidelines strikt, MNK sollten ,,not render - and they should not be solicited or expected to render - any bribe or other improper benefit, direct or indirect, to any public servant or holder of public office“. Mit dem Vorwand, saubere Geschäftspraktiken herstellen zu wollen, hoffen die USA offenbar, eine dreifache Zielvorstellung realisieren zu können. Erstens soll auf dieser freiwilligen Basis verhindert werden, daß MNK im Kielwasser der Lockheed-Affäre stärkerer nationaler Kontrolle unterworfen werden. Zweitens versuchen die USA davon abzulenken, daß hauptsächlich auf ihr Betreiben hin die OECD-Declaration in dem oben aufgezeigten Maß verwässert wurde ${ }^{32}$. Schließlich soll mit dieser Re-

30 Siehe ILM 1976, S. $977 \mathrm{ff}$.

31 Vgl. ,International Herald Tribune“ v. 27. 10. 75 und 26. 2. 76.

$32 \mathrm{Vgl}$. sehr deutlich hierzu ,Economist“" v. 26. 6. 76. 
duzierung der MNK-Problematik auf ein ethisch-verwerfliches Teilgebiet eine Ubertünchung der MNK-Tätigkeit erreicht werden. Zwar könnte eine verstärkte Bekämpfung von Bestechungen $u$. dgl. zur Verhinderung der Verzerrung von gleich gedachten Wettbewerbsbedingungen führen; die Begrenzung staatlicher Kontroll- und Regulierungsaktivitäten auf dieses Phänomen kann aber so der Gesamtproblematik nicht annähernd begegnen. Zudem dürfte die Umsetzung dieser Bestechungsverbote angesichts einer glänzend funktionierenden Korruptionsökonomie insbesondere in Entwicklungsländer realiter ohnehin auf erhebliche Widerstände stoßen. Die Vorschriften andererseits, die in den Guidelines in bezug auf die Informationsoffenlegung der MNK durch einen mindestens jährlich zu veröffentlichenden Bericht, dessen Inhalt konkret aufgeführt ist, formuliert wurden, sind zwar vergleichsweise detailliert - und insofern auch von MNK kritisiert worden ${ }^{33}$. Da die Einhaltung dieser Vorschriften aber ebenfalls nur freiwillig erfolgt, kann es nicht verwundern, daß letztere bislang kaum praktische Relevanz erhalten haben ${ }^{\mathbf{3 4}}$.

Sieht man die OECD-Declaration im Zusammenhang, so scheint sie die drei wesentlichsten Aufgaben, die Verhaltenskodizes aus der Perspektive der MNK selbst leisten sollen zu erfüllen $^{35}$. Zunächst soll öffentliche Kritik durch freiwillige Selbstregulierung begegnet werden. Weiterhin soll durch die Beschränkung auf ethisch-moralische Prinzipien von grundsätzlich strukturellen Problemen abgelenkt und zugleich durch die Stigmatisierung ,,schwarzer Schafe" unter den MNK ein Mindestmaß gleicher Konkurrenzbedingungen zwischen international operierenden Unternehmen gefördert werden. Zudem wird angestrebt, die von Entwicklungsländern intentionierte Stoßrichtung umzubiegen. Indem sich MNK zur Einhaltung solcher Kodizes verpflichten, sollen diese umgekehrt von den Gaststaaten quasi als langfristige Investitionsgarantieabkommen akzeptiert werden. So erwartet G. Tacke, ,,daß sich die OECD-Staaten Willkürhandlungen enthalten werden, sofern die Multinationalen Unternehmen die Guidelines befolgen“. In nuce werden folglich die Gaststaaten Adressaten derartiger Verhaltenskodizes.

Zur Durchsetzung dieser MNK-Interessen auch auf der UN-Ebene wird der OECD-Declaration eine Modellfunktion zugemessen. Dabei soll die Declaration bzw. die Guidelines insbesondere auf die Diskussion über die Formulierung eines universellen Verhaltenskodex, die im Rahmen der Commission on Transnational Corporations geführt wird ${ }^{\mathbf{3 6}}$, einwirken. Gerade hier allerdings bleibt die OECD-Declaration im Grunde auch aus der Sicht der MNK defizitär, da sie in der vorliegenden Form für Entwicklungsländer nicht akzeptabel ist. Ihre Modellfunktion kann sie somit nicht wahrnehmen, was wiederum negative Rückwirkungen auf die übrigen Funktionen hat ${ }^{37}$. Damit wird aber auch der Legitimationsbedarf der MNK also die eigentliche Kernfunktion dieser Declaration - nicht realisiert. Es bleibt abzuwarten, inwieweit diese Gesichtspunkte bei der wahrscheinlichen Teilrevision der OECD-Declaration, die nach drei Jahren praktischer Erprobung vorgesehen ist, berücksichtigt werden. Jedenfalls haben die vorerwähnten, weitergehenden und vor allem auf Rechtsverbindlichkeit abstellenden Kodifizierungsbemühungen eines MNK-Kodex im Rahmen der EG diese Kritikpunkte teilweise bereits aufgegriffen und verarbeitet.

\footnotetext{
33 Vgl. „Business Week“ v. 5. 4. 76.

34 Siehe "Blick durch die Wirtschaft" v. 22. 4. 78.

35 Siehehierzu und zum folgenden insbesondere G. Tacke (Aufsichtsratsmitglied der Siemens AG), Verhaltensregeln für Multinationale Unternehmen, in: Deutsch-Italienische Handelskammer (Hrsg.), ,, deutsch-italienischer handel“ 2/78. Ferner ,,Business Week“ v. 5. 4. 76 und ,Wall Street Journal" vom 28. 5. 76.

36 Darauf wird im nächsten Abschnitt ausführlich eingegangen.

37 Vgl. zu dieser Kritik auch ,, Nachrichten für Außenhandel“(NfA) v. 14. 7.76 und „Economist“"v. 26. 6. 76. Der ,,Economist“ beurteilt die Guidelines schlicht als ,,unnecessarily weak“.
} 
2.3. Regulierungsbestrebungen auf der UN-Ebene und den Konferenzen blockfreier Staaten

Die drei diskutierten Kodizes sollen insbesondere den Diskussionsprozeß in den Vereinten Nationen über MNK-Probleme beeinflussen. Der UNO kommen hierbei nicht nur als Fokus internationaler politischer Interessen wesentliche Diskussions- und Organisationsfunktionen zu, sondern sie dient darüber hinaus vor allem den Entwicklungsländern als Artikulationsforum, sowie faute de mieux als wichtigste kollektive Organisationsmöglichkeit und als ständiges Kommunikationszentrum. Insofern spielen die UN naturgemäß auch bei der Formulierung von MNK-Verhaltenskodizes eine wichtige Rolle. Im folgenden soll kurz aufgezeigt werden, inwieweit die Interessenpositionen der Ențicklungsländer in den UN Beachtung gefunden haben. Ausgeklammert werden die mit MNK nur mittelbar zusammenhängenden Problemfelder, wie die Verhaltenskodizes für Linienkonferenzen, gegen restriktive Geschäftspraktiken, Technologietransfer und Arbeitsbedingungen, für die z. T. auch schon Kodizes formuliert sind oder bei denen doch das Formulierungsprozedere zumindest weit fortgeschritten ist.

2.3.1. Resolutionen der UN und der Organisation blockfreier Staaten zur MNK-Problematik

Bestand in den 50er und 60er Jahren ein weitgehender Konsens über die vermeintlich positiven Entwicklungseffekte von MNK, so trat seitdem ein rapider Wandel in der Haltung der Entwicklungsländer zu diesem Problem ein. Zurückzuführen ist dieser Einstellungswandel einerseits auf die krisenhafte sozioökonomische Entwicklung des peripheren Kapitalismus sowie verschiedene fragwürdige Verwicklungen von MNK in die inneren Verhältnisse eines Gastlandes - Paradefall: die Interventionen von ITT in Chile - andererseits. Noch in der UN-Resolution zur zweiten Entwicklungsdekade vom Oktober 1970 wurden Direktinvestitionen in Entwicklungsländern äußerst positiv beurteilt ${ }^{38}$. So hieß es in Paragraph 50, auf den übrigens auch die ICC-Guidelines in deren Einleitung explizit rekurrierten, der do-utdes Haltung entsprechend, die Entwicklungsländer hätten einerseits geeignete Maßnahmen zur Heranziehung und Anwendung ausländischen Privatkapitals zu treffen sowie die Bedeutung eines günstigen Investitionsklimas in Rechnung zu stellen. Dafür sollten Industrieländer andererseits den Zustrom privater Investitionen in Entwicklungsländern fördern, und diese sich in nationale Entwicklungspläne einfügen. Außerdem wurde in allgemeiner Form eine bessere Einbeziehung und Qualifizierung einheimischer Arbeitskräfte, Beteiligung lokalen Kapitals und Gewinnreinvestition postuliert. Auf der anderen Seite wurde aber auch 1970 sowohl von den afro-asiatischen Staaten (auf der dritten Gipfelkonferenz blockfreier Staaten in Lusaka ${ }^{39}$ ) als auch den im Andenpakt integrierten lateinamerikanischen Staaten in der weiter unten diskutierten Entscheidung 24 die Relevanz von MNK für die Entwicklungsproblematik z. T. weniger, z. T. mehr erkannt. Weitergeführt wurden die in Lusaka begonnenen Anfänge auf der vierten Gipfelkonferenz blockfreier Staaten 1973 in Algier. In der wirtschaftlichen Erklärung wird u. a. in Abschnitt VIII zu MNK ausdrücklich auf die

38 UNGA-Res. 2626 (XXV). Abgedruckt in: K. Krakau/H. v. Wedel/A. Göhmann (Hrsg.), UN-General Assembly Resolutions, Frankfurt/M. 1975, S. $185 \mathrm{ff}$.

39 Zur dritten Gipfelkonferenz blockfreier Staaten in Lusaka siehe: Internationale Politik (Belgrad), Nr. $491 \mathrm{f}$. Ausführlich wird die positionelle Entwicklung der Haltung blockfreier Staaten zur MNK-Problematik dargestellt bei K. P. Sauvant, Controlling Transnational Enterprises: A Review and Some Further Thoughts, in: ders./H. Hasenpflug (Hrsg.), The New International Economic Order, Frankfurt/M. 1977, S. 389 ff. 
Souveränität der Entwicklungsländer sowie auf das Prinzip der Nichteinmischung und das Selbstbestimmungsrecht verwiesen ${ }^{\mathbf{4 0}}$. Gegenüber MNK sollen ferner konzertierte Maßnahmen der Mitgliedstaaten unternommen werden. Auf diese Maßnahmen geht das ebendort verabschiedete Aktionsprogramm für wirtschaftliche Zusammenarbeit detailliert $e^{4}{ }^{41}$.Danach sollen Direktinvestitionen und auch Reinvestitionen grundsätzlich genehmigungspflichtig gemacht werden. Diese Genehmigung wird davon abhängen, inwieweit diese Investitionen sich in nationale Entwicklungspläne einfügen, sich einer angepaßten Technologie bedienen, ,,zur Weiterentwicklung der Technologie beitragen, eine Quelle der Erwerbstätigkeit bilden, eine klare Einsparung an ausländischen Devisen darstellen, eine Verwaltung nach sich ziehen, die von der Muttergesellschaft unabhängig ist, u. a. “42. Restriktiv zu regeln sind ferner die Gewinnauszahlung im Konzern und der Aufkauf lokaler Unternehmen. Außerdem sollen ausländische Investitionen in strategischen Wirtschaftssektoren ausgeschlossen werden. Zwar haben somit die angesprochenen Staaten naturgemäß entsprechende Regeln zu erlassen, Hauptadressaten der Kritik aber sind unzweideutig die MNK. Mit diesen Vorschlägen konnten sich die Entwicklungsländer allerdings in den UN-Dokumenten zur Neuen Weltwirtschaftsordnung nicht voll durchsetzen. So wird in der Erklärung zur Errichtung einer Neuen Weltwirtschaftsordnung von $1974^{43}$ zwar ein Prinzip der „,Regulierung und Beaufsichtigung der Tätigkeit von multinationalen Gesellschaften durch Einleitung von Maßnahmen im Interesse der Volkswirtschaften derjenigen Länder, in denen derartige Gesellschaften operieren, auf der Grundlage der uneingeschränkten Souveränität dieser Länder"“44 aufgestellt. Im dazugehörigen Aktionsprogramm ist in Abschnitt V demgegenüber lediglich noch von allgemeiner Regulierung die Rede ${ }^{45}$. Darüber hinaus sollte hiernach bloß die Gewinnreinvestition von MNK in Entwicklungsländern gefördert und ihr Gewinntransfer ,, unter Berücksichtigung der legitimen Interessen aller betroffenen Seiten“ reguliert werden. Relativ unpräzise bleibt auch die Charta der wirtschaftlichen Rechte und Pflichten der Staaten, die zu dieser Frage in Art. 2 b) nur eine generelle Regulierungs-, Uberwachungs- und Kontrollkompetenz von Gaststaaten gegenüber MNK verlangt ${ }^{\mathbf{4 6}}$. Gleichwohl enthält die auf die Calvo-Doktrin verweisende Formel, wonach jeder Staat das Recht hat ,,die Tätigkeiten transnationaler Gesellschaften in seinem nationalen Hoheitsbereich zu regeln und zu überwachen und Maßnahmen zu treffen, um sicherzustellen, daß diese Tätigkeiten seinen Rechts- und sonstigen Vorschriften entsprechen und mit seinen wirtschaftlichen und sozialen Zielsetzungen in Einklang stehen“, in nuce ein Essential lateinamerikanischer Völkerrechtsdoktrin ${ }^{47}$. Ergänzend wird ein Interventionsverbot für MNK in die inneren Angelegenheiten von Gaststaaten gefordert. Im Zusammenhang mit dem in demselben Artikel sehr dezidiert verankerten Prinzip ständiger Souveränität über natürliche Ressourcen betrachtet, gewinnen diese Bestimmungen stark an Bedeutung. Insofern messen die Entwicklungsländer der Charta auch in bezug auf zu formulierende Verhaltenskodizes für MNK grundsätzliche Bedeutung $\mathrm{zu}^{\mathbf{4 8}}$.

\footnotetext{
40 Text in: Internationale Politik (Belgrad), Nr. 563. Auszugsweise auch in: Europa-Archiv 1973, S. D 580 ff.

41 Text auch in: Blätter für deutsche und internationale Politik 1974, S. $311 \mathrm{ff}$.

42 Ebd.

43 UN-Res. 3201 (S.-VI). Abgedruckt in: ebd., S. 639 ff.

44 Ebd., Abschnitt IV g).

45 UN-Res. 3202 (S.-VI). Abgedruckt in: ebd., S. 643 ff. und $753 \mathrm{ff}$

46 UNGA-Res. 3281 (XXIX). Abgedruckt in: Bundesministerium für wirtschaftliche Zusammenarbeit (Hrsg.), Materialien Nr. 49, S. $11 \mathrm{ff}$.

47 Vgl. ausführlich hierzu: K. Krakau, Lateinamerikanische Doktrinen zur Realisierung staatlicher Unabhängigkeit und Integrität, in: Verfassung und Recht in Ubersee (VRU) 1975, S. $117 \mathrm{ff}$.

48 ,It is vital that the future code of conduct clearly reflect the Charter's postulates", „Comercio de México" 5/75.
} 
Enthalten die genannten Dokumente zur neuen Weltwirtschaftsordnung in bezug auf MNK zumindest die zentralen Forderungen der Dritten Welt, so konnte letztere sich in dieser Frage auf der zweiten Generalkonferenz der UNIDO 1975 nicht mehr durchsetzen. Zurückzuführen ist dies im wesentlich auf ihre verschlechterte ökonomische Ausgangsposition. Während die OOlpreiserhöhung der OPEC die Verhandlungsmacht der Entwicklungsländer zunächst stärkte, und diese verbesserte Position gerade auch auf die Beratungen über die neue Weltwirtschaftsordnung ausstrahlte, führte die nachfolgende weltweite wirtschaftliche Rezession, die insbesondere viele kleinere und mittlere Entwicklungsländer betraf, zur Schwächung dieser Position. Dies schlug sich bereits in Erklärung und Aktionsplan über industrielle Entwicklung und Zusammenarbeit der erwähnten UNIDO-Konferenz nieder ${ }^{49}$. So wird in Paragraph 42 zur Eindämmung anarchischer Penetration des industriellen Sektors der Entwicklungsländer durch MNK lediglich eine allgemein formulierte Regulierung und Úberwachung von MNK gefordert. Paragraph 58, der Elemente einer nationalen Industrialisierungspolitik enthält, gibt nur mittelbar Aufschluß über die ökonomischen Voraussetzungen, die für eine sinnvolle Mitwirkung von MNK betreffs der Erfüllung nationaler Entwicklungspläne erfüllt sein sollten. Außerdem fordert Paragraph 59 Absätze g) und h) die Industrieländer dazu auf, ihre MNK zu dieser Mitwirkung anzuhalten. Auch die siebte Sondersitzung der UN und UNCTAD IV haben der Diskussion über diese Frage aus dem genannten Grund keine neuen Impulse verliehen. Zudem konzentrierten sich die Entwicklungsländer auf diesen Konferenzen primär auf Handelsprobleme ${ }^{50}$.

Kontrovers zwischen Industrie- und Entwicklungsländern blieb das Thema MNK auch auf der ILO-Weltbeschäftigungskonferenz 1976; ein Konsensus wurde nicht erzielt. Statt dessen gab jede Gruppe - gemäß der triparitären Struktur der ILO auch Unternehmer und Gewerkschaften - getrennte Voten $\mathrm{bb}^{\mathbf{5 1}}$. Dabei ist bemerkenswert, daß die von der Gruppe der 77 - also den Entwicklungsländern - in Paragraph 85 dieser ILO-Prinzipienerklärung verwendeten Formulierungen praktisch wörtlich identisch sind mit einem Positionspapier, das zehn lateinamerikanische Staaten in die Diskussion der Commission on Transnational Corporations eingebracht hatten ${ }^{52}$. Die in Paragraph 85 enthaltenen elf Grundsätze bilden die Grundprinzipien, die ein MNK-Verhaltenskodex aus der Sicht der Entwicklungsländer beinhalten sollte: , ,1. TNCs must be subject to the laws and regulations of the host country and in the event of a dispute accept the exclusive jurisdiction of the courts of the country in which they operate; 2 . TNCs should refrain from all interference in the internal affairs of the States in which they operate; 3 . TNCs should refrain from interference in their relations between the government of the host country and other States, and from influencing these relations; 4. TNCs should not serve as an instrument of the external policy of another State nor as a means of extending to the host country juridical regulations of the country of origin; 5 . TNCs should be subject to the permanent sovereignty which the host country exercises over all its wealth, natural recources and economic activities; 6. TNCs should comply with national development policies, objectives and priorities and make a contribution to their im-

49 Abgedruckt in BMZ-Materialien Nr. 49, S. $31 \mathrm{ff}$.

50 Ahnlich verhält es sich mit dem zwischen den AKP-Staaten und der EWG abgeschlossenen Abkommen von Lome: Konzentrierten sich die Entwicklungsländer hauptsächlich auf die Durchsetzung ihrer handelspolitischen Forderungen, so waren sie andererseits gegenüber der EWG in sekundären Fragen durchaus zu Konzessionen bereit. Wenn sich die A KP-Staaten etwa in Art. 38 zur Schaffung eines günstigen Investitionsklimas verpflichten, ist dies nicht per se Ausdruck eines Gesinnungswandels, sondern reflektiert eher den Kompromißcharakter des Lomé-Abkommens. Vgl: hierzu: R. Tetzlaff, Die Forderungen der Entwicklungsländer nach einer ,,Neuen" Weltwirtschaftsordnung, die Internationalisierung der Produktion und das AKP-Abkommen von Lomé, in: VRU 1976, S. 33 ff.; K. Unger, Die EG und die Entwicklungsländer. Das Abkommen von Lomé als Grundstein einer neuen Weltwirtschaftsordnung? in: Blätter für deutsche und internationale Politik 1977, S. $301 \mathrm{ff}$.

51 Siehe Declaration of Principles, in: World Employment Conference, Geneva, 4-17 June 1976, Summary Record 20, Abschnitt V.

52 Areas of Concern which could be Used as a Basis for Preliminary Work for a Code of Conduct to be Observed by Transnational Corporations (E/C. 10/L. 2); abgedruckt in: E/5782, a. a. O., S. $27 \mathrm{ff}$. 
plementation; 7. TNCs should supply the government of the host country with relevant information on their activities in order to ensure that those activities are in accordance with the national development policies, objectives and priorities of the host country; 8. TNCs should conduct their operations in such a way that they result in a net inflow of financial resources for the host country; 9. TNCs should contribute to the development of the domestic, scientific and technological capacity of the host countries; 10. TNCs should refrain from restrictive trade practices; 11 . TNCs should respect the sociocultural identity of the host country ${ }^{53}$." Auffallend sind die strikten, wenn auch nur knappen Formulierungen, die ausschließlich an die Adresse der MNK gerichtet sind. Auffallend ist weiterhin die erneute enge Verflechtung von Calvo-Doktrin mit dem Prinzip ständiger Souveränität über natürliche Ressourcen, dessen enteignungsvölkerrechtlicher Kern in der Verstaatlichungsmöglichkeit ausländischen Eigentums nach nationalem Recht besteht ${ }^{54}$. Paragraph 85 der Prinzipienerklärung kommt insbesondere auch deshalb Bedeutung $\mathrm{zu}$, weil das weitergehende MNKStatut der blockfreien Staaten nicht die ursprünglich intendierte internationale Ausformung erhalten hat ${ }^{55}$. Da die sozioökonomische und politische Basis der einzelnen Entwicklungsländer zu unterschiedlich war, um das Statut auf einergemeinsamen Grundlage international anzuwenden, wurde es nicht in der geplanten Form angenommen. Damit bleibt die Umsetzung und Anwendung dieses Statuts den jeweiligen Staaten selbst überlassen. Die ILO-Prinzipienerklärung könnte in diesem Punkt die Funktion eines Grundkonsenses für weitergehende Aktionen der Entwičklungsländer übernehmen. Zusammenfassend läßt sich feststellen, daß einerseits die Ansätze für einen MNK-Verhaltenskodex in der UN-Resolutionspraxis noch fragmentarisch geblieben sind. Andererseits sind aber bereits einige wesentliche $\mathrm{Ba}$ sisprinzipien von den Entwicklungsländern entwickelt worden, die einen generellen Konsens über die von MNK zu gewährleistenden Verhaltensweisen bei der Mitwirkung an der Erfüllung nationaler Entwicklungspläne bilden.

\subsubsection{Die Beratung in der UN-Kommission für transnationale Konzerne}

Im Rahmen der Tätigkeit der UN-Commission on Transnational Corporations soll erstmalig der Versuch gemacht werden, auf internationaler Ebene einen universell geltenden und thematisch umfassenden Verhaltenskodex für MNK auszuarbeiten ${ }^{56}$. Die oben aufgezeigten Interessenkonstellationen der beteiligten Akteure kommen auch in den in der Commission geführten Beratungen zur Geltung. Den direkten Anstoß zur Bildung einer UN-Kommission für MNK gab die ITT-Intervention in Chile. Nachdem diese bekannt geworden war, regte Chile unterstützt von Mexiko 1972 an, im Rahmen der UN eine Expertengruppe zu etablieren, die die mit der Tätigkeit von MNK in Gastländern verbundenen Probleme unter-

53 Declaration of Principles, a. a. O.

54 Vgl. hierzu auch die in E/C. 10/L. 2 enthaltenen Erläuterungen. Ausführlich zum Prinzip ständiger Souveränität über natürliche Ressourcen: G. Brehme, Souveränität junger Nationalstaaten über Naturreichtümer, Berlin (DDR) 1967; G. Hartmann, Nationalisierung und Enteignung im Völkerrecht, Berlin 1977; ders., Selbstbestimmung, Unabhängigkeit und Gewaltverzicht. Die Beziehungen der industrialisierten kapitalistischen Nationen zu den Entwicklungsländern und das Völkerrecht, in: Demokratie und Recht $1975, \mathrm{~S}$. $248 \mathrm{ff}$. Eine Veröffentlichung d. Verf. zum Thema befindet sich in Vorbereitung.

55 Ausführlich hierzu: Sauvant, Controlling ..., a. a. O.

56 Im folgenden sollen nur einige wesentliche Grundzüge und Probleme der Tätigkeit der Commission aufgezeigt werden, da zur Problematik schon eine Vielzahl von Veröffentlichungen erschienen ist. Vgl. u. a.: K. W. Grewlich, Gegenwärtiger Stand der Arbeiten in der UN-Kommission für transnationale Unternehmen, in: Recht der Internationalen Wirtschaft/Außenwirtschaftsdienst des Betriebs-Beraters (RIW/AWD) 1976, S. 389 ff.; S. J. Rubin, Reflections concerning the United Nations Commission on Transnational Corporations, in: American Journal of International Law (A JIL) 1976, S. 73 ff.; N. T. Wang, The International Community and Transnational Corporations, in: K. P. Sauvant/F. J. Lavipour (Hrsg.), Controlling Multinational Enterprises, Frankfurt/M. - New York 1976, S. 215 ff.; R. Hellmann, Die Kontrolle multinationaler Unternehmer, Baden-Baden 1974, S. 67 ff.; ,,CTC-Reporter“, No. $1 \mathrm{ff}$. 
suchen sollte. Diese Anregung wurde in einer Resolution des UN-Wirtschafts- und Sozialrats (ECOSOC) im Juli 1972 aufgegriffen, wobei die zu bildende Gruppe insbesondere die Rolle der MNK im Entwicklungsprozeß von Entwicklungsländern und in den internationalen Beziehungen analysieren sollte ${ }^{57}$. Unterstützt wurde die Arbeit dieser Expertengruppe, die zwischen September 1973 und April 1974 dreimal zusammentraf, durch einen MNKReport des UN-Sekretariats ${ }^{58}$. Kapitel IV dieses Reports enthält bereits Aktionsvorschläge für ein internationales Programm zur MNK-Problematik. Danach sollte im Rahmen des ECOSOC ein Forum zur Diskussion dieser Problematik geschaffen werden, das von einem Informationszentrum, das anfallende Informationen über MNK zusammentragen und aufarbeiten soll, zu unterstützen wäre ${ }^{59}$. Außerdem wurde eine technische Zusammenarbeit mit entsprechenden Behörden der Entwicklungsländer vorgeschlagen, um deren Verhandlungsmöglichkeiten gegenüber MNK zu verbessern. Skeptisch wird zum gegenwärtigen Zeitpunkt die Möglichkeit zur Verwirklichung eines bindenden Allgemeinen Abkommens für MNK beurteilt. Andererseits wird aber einem unterhalb dieser Ebene bleibenden weniger verbindlichen Verhaltenskodex, der im ECOSOC ausgearbeitet werden könnte, ein zumindest ,erzieherischer Wert" zugebilligt ${ }^{60}$.

Diese Empfehlungen und Einschätzungen sind auch im Report der Experten (Group of Eminent Persons) aufgegriffen worden ${ }^{\mathbf{6 1}}$. In der wichtigsten Empfehlung der Group wird die Bildung einer dem ECOSOC angegliederten Commission für MNK gefordert ${ }^{62}$. Diese sich aus 25 unabhängigen Experten zusammensetzende Commission on Multinational Corporations hätte danach als allgemeines Forum zu dienen, zugleich aber auch angemessene Aktions- und Arbeitsprogramme zu entwerfen sowie als Basis weitergehender Maßnahmen zu fungieren. Außerdem empfahl die Group ebenfalls die Bildung eines dem UN-Sekretariat angeschlossenen Information and Research Centre on Multinational Corporations, das die Commission durch einen Informations- und Forschungsdienst in ihrer Arbeit unterstützen sollte. Ebenfalls betont wurde die technische Zusammenarbeit mit Entwicklungsländern ${ }^{\mathbf{6}}$. Die Group teilt die Skepsis gegenüber einem kurzfristig verwirklichbaren Allgemeinen MNK-Abkommen. Längerfristig allerdings wird empfohlen, das Ziel eines Allgemeinen Abkommens über MNK mit der Bindungswirkung eines internationalen Vertrags und Ausführungs- und Sanktionsbestimmungen im Auge zu behalten ${ }^{64}$. Da dies aber im Moment verfrüht wäre, sollen über verschiedene Einzelelemente Arbeiten weitergeführt werden, die sukzessive auf ein umfassendes, international bindendes Abkommen hin extrapoliert werden können. Ein Verhaltenskodex bestünde dann zunächst nur aus einem Bündel diverser (rechtlich unverbindlicher) Einzelempfehlungen, die in ihm integriert wären ${ }^{65}$. Diesen dermaßen definierten Verhaltenskodex, der sowohl für Staaten als auch MNK gelten würde, auszuarbeiten, sollte in den Tätigkeitsbereich der Commission fallen ${ }^{66}$. Die Group selbst hat bereits Empfehlungen für verschiedene Einzelbereiche formuliert: Eigentumsstruktur und Kontrolle, Kapitalströme und Zahlungsbilanz, Technologie, Beschäftigung und Arbeit, Verbraucherschutz, Wettbewerb und Marktstruktur, Transferpreissystem, Besteuerung und Rechnungslegung. Dabei fällt auf, daß diese Empfehlungen nicht nur an die Adresse der

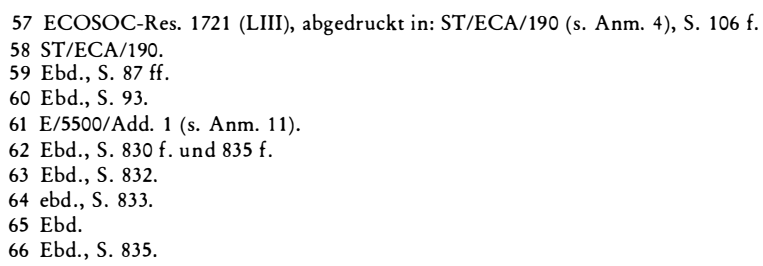


MNK, sondern auch die der (Gast-)Staaten gerichtet sind. Unmittelbar einsichtig ist natürlich, daß MNK sich nur dann an ökonomische Rahmenpläne halten können, wenn diese überhaupt von den Gaststaaten formuliert worden sind. Gleichwohl impliziert dieses Vorgehen der Group letztlich die Fiktion einer vollständigen Waffengleichheit der beteiligten Akteure, deren reale Macht- und Größenverhältnisse dadurch verunklart werden. Das zentrale Anliegen der Group, die Stärkung der Verhandlungsposition der Entwicklungsländer gegenüber MNK, könnte hierdurch relativiert werden.

Vom ECOSOC wurden die Empfehlungen der Group of Eminent Persons hinsichtlich der Schaffung einer Commission und eines Informations- und Forschungszentrums über MNK-Fragen aufgegriffen. Nachdem in ECOSOC-Res. 1913 (LVII) die Bildung der Commission entschieden wurde ${ }^{67}$, fand deren erste Sitzung im März 1975 statt und seitdem turnusgemäß in jedem Frühjahr ${ }^{68}$. Anders als von der Group vorgeschlagen, umfaßt die Commission allerdings 48 Mitglieder, die überdies keine unabhängigen Experten, sondern weisungsgebundene Vertreter von Staaten sind. Uber die Etablierung des Centre wurde in ECOSOC-Res. 1908 (L VII) beschlossen ${ }^{69}$. Das Centre arbeitet seit Herbst $1975^{70}$.

Das Arbeitsprogramm der Commission, das von der besonderen Zielvorstellung der Erhöhung der ,,negotiating capacity“ von Entwicklungsländern ausgeht, umfaßt folgende Sachgebiete: 1. Formulierung eines MNK-Verhaltenskodex; 2. Aufbau eines umfassenden Informationssystems; 3 . Forschung über politische und sozioökonomische Auswirkungen der MNK-Aktivitäten; 4. Technische Koordination, 5. Definition von MNK. Wurden auf der ersten Session der Commission die zwischen Entwicklungs- und Industrieländern bestehenden Kontroversen noch sichtbar, so blieb die für die zweite Session erwartete Konfrontation aus, da strittige Probleme weitgehend ausgeklammert wurden ${ }^{71}$. Der tiefere Grund liegt auch hier in dem abnehmenden Verhandlungsspielraum der Entwicklungsländer. Insofern brachten auch die dritte Session 1977, die hauptsächlich einem Informationsaustausch diente, und die vierte Session 1978 nur partielle Fortschritte ${ }^{72}$.

An das Arbeitsprogramm der Commission lehnt sich seinen Aufgaben entsprechend die Arbeit des Centre an ${ }^{73}$. Im Vordergrund steht dabei der Aufbau eines umfassenden Informationssystems ${ }^{\mathbf{7 4}}$, mit dem die Entwicklung international akzeptierter Bilanzierungsstandards korrespondieren. Darüber hinaus soll auch ein Maßnahmenkatalog zur Verhinderung von Bestechungspraktiken erarbeitet werden. Diese sechs Arbeitsbereiche zusammengenommen, verdeutlichen die enorme Arbeitsbelastung, der das Centre unterliegt.

$\mathrm{Zu}$ den umstrittensten Themen zählt die Problematik von Informations- bzw. Bilanzierungssystemen, die beide wesentliche Elemente eines Verhaltenskodex' bilden. In bezug auf die Informationserhebung durch das Centre ${ }^{75}$ sind es insbesondere verschiedene Industrie-

\footnotetext{
67 Abgedruckt in: Economic and Social Council, Official Records, 57th Session, Resolutions Suppl. No. 1A, New York 1975 (E/5570/Add. 1).

$68 \mathrm{Daß}$ sich die Bezeichnung der Commission von Commission on Multinational Corporations in Commission on Transnational Corporations geändert hat, geht auf einen Vorschlag der Group zurück, die mit dem Ausdruck ,,transnational“ den grenzüberschreitenden Charakter der MNK-Tätigkeit verdeutlichen wollte; a. a. O., S. 807, Anm. 2.

69 Abgedruckt in: ebd. (Anm. 67), Suppl. No. 1, New York 1974 (E/5570).

70 Die Verkürzung der Bezeichnung von Information - and Research Centre on TNCs zu Centre on TNCsberuht darauf, daß die Tätigkeit des Centre mehr als nur Informations- und Forschungsdienste zu gewährleisten hat.

71 Siehe United Nations, Commission on Transnational Corporations, Report on the first session. Official Records of the Economic and Social Council, 59th Session, Suppl. No. 12 (E/5655); United Nations, Commission on Transnational Corporations, Report on the second session. Official Records. of the Economic and Social Council, 61st Session, Suppl. No. 5 (E/5782); NfA v. 22. 3. 76.

72 Vgl. United Nations, Commission on Transnational Corporations, Report on the resumed 2nd session and the 3rd session. Official Records of the Economic and Social Council, 63rd Session Suppl. No. 5 (E/5986); NZZ v. 19. 5. und 28. / 29. 5. 78.

73 Siehe hierzu insbesondere: United Nations, Commission on Transnational Corporations, Statement of the Executive Director on the Activities of the Centre on Transnational Corporations Presented at its 23rd Meeting (27. 4. 77), (E/C. 10/30).

74 Hiermit im Zusammenhang steht auch die Herausgabe eines Periodikums, des ,,CTC-Reporter“, von dem bislang vier Nummern erschienen.

75 Vgl. United Nations, Commission on Transnational Corporations, Development of a Comprehensive Information System on Transnational Corporations: Report of the Secretariat (E/C. 10/41).
} 
staaten, denen die Aktivitäten des Centre zu weit gehen, da es mehr Informationen ermittelt und veröffentlicht als in den jeweiligen Staaten zulässig ist ${ }^{\mathbf{7 6}}$. Da aber diese zentrale Institution zur internationalen Informationserfassung über MNK ja gerade nationalstaatliche Kirchturmspolitik überwinden will, scheint dieser Einwand nur bedingt stichhaltig. Aus der Sicht der Entwicklungsländer erscheint ein derartiger UN-Informations- und Forschungspool überdies als Gegengewicht zu MNK. Denn was einzelne Entwicklungsländer kaum realisieren können - nämlich Schaffung größerer Transparenz - kann hier auf kollektiver Ebene durch das Centre, das darin ihrer Meinung nach seine wesentlichste Aufgabe erfülltt ${ }^{77}$, in Form von Sammlung und Verfügbarmachung relevanter MNK-Informationen erreicht werden. Die vorgeschlagenen Bilanzierungsformen ${ }^{78}$ werden demgegenüber besonders von MNK attackiert, die vor allem um die Vertraulichkeit von Informationen fürchten ${ }^{79}$. Insofern werden hier nur erneut Einwände geäußert, die bereits kritisch gegenüber den OECD-Guidelines erhoben wurden. Da überdies die internationalen Bilanzierungsvorschriften für alle wichtigen Unternehmen gleichermaßen gültig sein werden, ist auch eine einseitige Verschlechterung der Wettbewerbsposition nicht zu erwarten. Gleichwohl scheinen sich momentan die Auseinandersetzungen in der Commission auf dieses Problem zu konzentrieren.

Priorität hat die Commission der Formulierung eines MNK-Verhaltenskodex' gegeben. Ein Entwurf hierzu wurde in einer zu diesem Zweck gebildeten zwischenstaatlichen Arbeitsgruppe beraten, wobei außerdem das Centre einen aus 181 Paragraphen bestehenden Problemkatalog in bezug auf MNK-Kodizes vorlegte ${ }^{\mathbf{8 0}}$. Die von Industrie- und Entwicklungsländern verfolgten unterschiedlichen Konzeptionen treten in der Arbeit der Commission zu dieser Frage deutlich hervor. Das zeigt sich schon in der Auseinandersetzung darüber, auf welche UN-Resolutionen sich die Commission als praktischer Anknüpfungspunkt beziehen soll. Während die meisten Entwicklungsländer die einschlägigen UN-Dokumente zur Schaffung einer neuen Weltwirtschaftsordnung als Bezugspunkt ansehen, was logisch und sachlich einleuchtend erscheint, da Veränderungen im Verhältnis von Entwicklungsländern zu MNK einen integralen Bestandteil der Umstrukturierung weltwirtschaftlicher Prozesse bilden werden, berufen sich zahlreiche Industrieländer auf deren nach wie vor bestehende Ablehnung dieser Resolutionen. Umstritten ist auch die Definition von MNK. Diese soll nach dem Willen der Industrieländer auch staatliche sowie in Entwicklungsländern ansässige multinationale Unternehmen umfassen. Da sich für diese Unternehmen aber das Problem von Transparenz, Kontrolle und gesellschaftlicher Partizipation keinesfalls in gleicher Weise stellt, wie für die in den westlichen Industrieländern ansässigen MNK, lehnen die Entwicklungsländer eine derartig weitgefaßte Definition ab. Da sich in dieser Frage kein Konsensus erzielen ließ, wurde die Definitionsproblematik zunächst aus den Beratungen der Commission ausgeklammert.

\footnotetext{
76 Vgl. Grewlich, Gegenwärtiger Stand . . ., a. a. O., S. 394

77 Vgl. J. D. Akumu, The importance of the Centre to developing countries, in: „CTC-Reporter“ $2 / 77, \mathrm{~S} .17 \mathrm{f}$.

78 United Nations, Commission on Transnational Corporations, International Standards of Accounting and Reporting for Transnational Corporations, New York 1977, (E/C. $10 / 33)$.

79 Vgl. „Financial Times“ v. 7. 4. 78.

80 Siehe United Nations, Commission on Transnational Corporations, Work Related tothe Formulation of a Code of Conduct. Report of the Intergovernmental Working Group on the Code of Conduct (4. 5. 77), (E/C. 10 / 31); United Nations, Centre on Transnational Corporations, Transnational/Corporations: Issues Involved in the Formulation of a Code of Conduct (E/C. 10/17). Mit der letztgenannten Studie in engem Zusammenhang stehen die Materialien zu nationalen und internationalen Regulierungsversuchen für MNK: United Nations, Commission on Transnational Corporations, National Legislation and Regulations Relating to Transnational Corporations (E/C. 10 / 8 / Add. 1); United Nations, Commission on Transnational Corporations, International Codes and Regional Agreements Relating to Transnational Corporations (E/C. 10 / 9 and Add. 1); United Nations, Centre on Transnational Corporations, Transnational Corporations: Material Relevant to the Formulation of a Code of Conduct (E/C. 10/18).
} 
Auch in bezug auf andere inhaltliche Probleme läßt es der Katalog des Centre ${ }^{\mathbf{8 1}}$ mit dem Skizzieren möglicher Lösungen bewenden. Als Adressaten werden allerdings analog zu dem Report der Group of Eminent Persons sowohl Heimat- und Gaststaaten, als auch MNK genannt. Inhaltliche Formulierungen sind aber aufgrund der einander z. T. diametral entgegengesetzten Optionen der beteiligten Akteure selten fixiert. So fordert etwa die Gruppe der Industriestaaten vor allem einen verbesserten Schutz von MNK gegenüber Interventionen des peripheren Staates ${ }^{\mathbf{8 2}}$, während demgegenüber die Gruppe der 77 primär negative politische und ökonomische Effekte von MNK bezogen auf die Situation der Entwicklungsländer in den Vordergrund rücken ${ }^{83}$. Besonders kontrovers ist auch hier das Prinzip der ständigen Souveränität über natürliche Ressourcen. Dabei ist von Entwicklungsländern kritisiert worden, daß dieses für sie essentielle Völkerrechtsprinzip weder im Formulierungsvorschlag der zwischenstaatlichen Arbeitsgruppe noch im Katalog des Centre adäquat reflektiert wurde ${ }^{84}$. Offen ist ferner die endgültige Formulierungs- bzw. Durchsetzungsweise des geplanten Kodex' sowie dessen Rechtscharakter, die ebenfalls kontroverse Beratungsgegenstände sind ${ }^{\mathbf{8 5}}$. In dieser Frage konnten sich die Staatengruppen lediglich auf die verschwommene Kompromißformel eines zu formulierenden ,,effektiven“ Verhaltenskodex einigen. Mithin hat sich weder eine - im eingangs genannten Sinn - maximalistische noch eine minimalistische Konzeption durchgesetzt. Wenn man davon ausgeht, daß ein effektiver Verhaltenskodex zumindest eine konkrete sprachliche Gestaltung impliziert, bleibt andererseits die Frage des legal binding und die der Streitschlichtung offen. In der umstrittenen Frage der Rechtsverbindlichkeit des MNK-Kodex' könnten sich allerdings auf der Basis der oben skizzierten Entwicklungen eines europäischen Kodex' Kompromisse erzielen lassen. Die insbesondere von den Gewerkschaften vorgetragene Kritik an den OECD-Richtlinien, letztere seien im Grunde nur ,,zahnlose“ Grundsatzerklärungen, scheint hier bereits Früchte getragen zu haben. Falls es insofern gelingt, auch den angestrebten UN-Kodex auf ein rechtsverbindliches Fundament zu stellen, wäre damit ein erster Schritt über ein bloßes ,,instrument of moral persuasion " hin auf ein Allgemeines Abkommen über Direktinvestitionen getan ${ }^{86}$. Damit ist auch das Problem der Einklagbarkeit bzw. Sanktionierbarkeit angesprochen. Während die oben aufgezeigte maximale Formulierungslösung implizit davon ausging, daß MNK zum alleinigen Adressaten des Verhaltenskodex' werden, sind in den genannten Entwürfen auch die (Gast-)Staaten angesprochen. Insoweit aber auch diese Staaten in den Kodex mit einbezogen werden, ergibt sich für Entwicklungsländer eine ambivalente Situation, da sie in einer Internationalisierung möglicher Rechtsstreitigkeiten sowohl eine Beschneidung ihrer staatlichen Souveränität als auch eine argumentative Unterlegenheit gegenüber MNK, die gegenwärtig noch über ein weitgehendes Informationsmonopol verfügt, befürchten. Dieser Ambivalenz könnte durch eine Doppelkonstruktion Rechnung getragen werden, die es ermöglicht, daß einerseits MNK, vor einen zu schaffenden internationalen Investitionsge-

\footnotetext{
81 E/C. $10 / 17$.

82 ,Areas of Concern which Relate to Relations Between Transnational Corporations and Governments“, abgedruckt in: E/5655, S. 20 ff.

83 „List of Areas of Concern Regarding the Operations and Activities of Transnational Corporations“, abgedruckt in: ebd., S. $18 \mathrm{f}$.

$84 \mathrm{Vgl}$. E/C. $10 / 31$, S. 3. Anzumerken bleibt selbstverständlich, daß die Entwicklungsländer durchaus nicht immer als quasi monolithischer Block auftreten. So scherte bsplw. Argentinien aus der Gruppe lateinamerikanischer Staaten aus, indem es zwecks Stimulierung größerer Direktinvestitionen selbst ein für diese Staaten so fundamentales Völkerrechtsprinzip wie die Calvo-Doktrin aufgab. Vgl. hierzu: ,Journal of Commerce“ v. 9. 3. 78.

$85 \mathrm{Vgl}$. Developing and Enforcing Guidelines for Multinational Corporations, Remarks by K. A. Sahlgren, in: „,Proceedings of the American Society of International Law" 1976, S. 27

$86 \mathrm{Vgl}$. E/5500/Add. 1, S. 833.
} 
richtshof zitiert werden können, andererseits aber Klagen von MNK gegen Gaststaaten nur von den jeweiligen Gerichten der letzteren behandelt werden dürfen. Wie allerdings die endgültige Lösung dieses Problems aussehen wird, läßt sich zur Zeit noch nicht absehen ${ }^{87}$.

\section{Regionale und nationale Investitionskodizes}

Stark beeinflußt worden ist die internationale Diskussion über MNK-Kodizes von den Direktinvestitionsbestimmungen des Andenpakts, von denen einige relevante im folgenden kurz skizziert werden sollen. Grundlage der Behandlung von Auslandskapital sind für die meisten Entwicklungsländer nationale Investitionsgesetze. Paradigmatisch soll daher anschließend auf den neuen Investitionscode Vietnams eingegangen werden.

\subsection{Die Entscheidung 24 des Andenpakts}

Ein essentieller Bestandteil ökonomischer Integrationen in der Dritten Welt müssen Regulierungsvorschriften für MNK sein. Zum einen gilt es auf dem Wege der Harmonisierung nationaler Investitionsgesetze einen regionalen ,,scramble for investment " zu verhindern und so zugleich die Verhandlungsmacht gegenüber MNK zu erhöhen. Parallel hierzu müssen zum anderen inländische Unternehmen gestärkt werden, um zu vermeiden, daß nur MNK von der Integration profitieren, die aufgrund ihrer Faktorausstattung eher auf diese Weise ermöglichte economies of scale realisieren können.

Von diesen Úberlegungen geht auch das Lima-Statut (= Entscheidung 24) des Andenpakts aus $^{88}$. Die wichtigsten Bestimmungen der Entscheidung 24 lassen sich in fünf Punkten zusammenfassen ${ }^{89}$. Primär wird die Umwandlung von in der Region operierenden MNK in inländische bzw. gemischte Unternehmen angestrebt. Dabei sind inländische Unternehmen definiert durch einen Anteil des Inlandskapitals von mindestens $80 \%$ und gemischte durch einen entsprechenden Anteil von $51-80 \%$. Übrige Unternehmen gelten als ausländische MNK. Für die Umwandlung wird eine Frist von höchstens 15 Jahren eingeräumt (für Bolivien und Ecuador 20 Jahre). Sollten einige MNK diese Bestimmung nicht befolgen, so sieht das Lima-Statut als Sanktion vor, daß diese MNK nicht an den Integrationsvorteilen der angestrebten regionalen Zollunion partizipieren dürfen. Ausgenommen von dieser Vorschrift werden u. a. MNK, die mehr als $80 \%$ ihrer Produkte in Drittländer exportieren. Weiterhin werden MNK aus bestimmten Produktions- und Dienstleistungssektoren (Finanzinstitute, öffentliche Dienstleistungen, Medien, Werbung, Transport) gänzlich ausgeschlossen. Drittens wird der Gewinntransfer von MNK auf $14 \%$ p. a. bezogen auf das investierte Gesamtkapital begrenzt. Ferner ist die Aufnahme von langfristigen lokal finanzierten Krediten für MNK ausgeschlossen. Außerdem sollen verschiedene Vorschriften restriktive Geschäfts-

\footnotetext{
87 Vgl. NZZ v. 7. 10. 78 und v. 23. 1. 1979

88 Textentwurf in: ILM 1971, S. $152 \mathrm{ff}$; endgültige Fassung in: ILM 1972, S. $126 \mathrm{ff}$

89 Es mag an dieser Stelle genügen, den Inhalt dieser Entscheidung 24 und dessen Problematik nur grob zu skizzieren, da hierzu bereits zahllose Untersuchungen vorliegen. Vgl. etwa: C. T. Oliver, The Andean Foreign Investment Code: A New Phase in the Quest for Normative Order as to Direct Foreign Investment, in: AJIL 1972, S. 763 ff.; K. Matter, Die Bestimmungen des Andenpaktes über die gemeinsame Behandlung des Auslandkapitals, in: Außenwirtschaft 1972, S. 56 ff.; D. B. Furnish, The Andean Common Market's Common Regime for Foreign Investments, in: K. P. Sauvant/F. J. Lavipour (Hrsg.), Controlling . . . a. a. O. (Anm. 56), S. 181 ff.; D. Boris/A. Frambes-Alzérreca, Der Andenpakt. Ein Bündnis gegen Unterentwicklung und Vorherrschaft ausländischen Kapitals, in: Blätter für deutsche und internationale Politik 1972, S. 1172 ff.; Schlupp, a. a. O. (Anmerkung 13), S. 206 ff.; Sauvant, a. a. O. (Anm. 39), S. 366 ff.
} 
praktiken und exorbitante Transferpreise verhindern. In diesem Zusammenhang ist schließlich relevant, daß ausländische Investitionen und Reinvestitionen grundsätzlich genehmigungspflichtig sind. Keine Investitionsgenehmigung wird erteilt, wenn der Markt schon durch bestehende Unternehmen ausreichend versorgt wird. Der Aufkauf inländischer Firmen durch MNK ist nur im Fall des Bankrotts ersterer gestattet.

Mit diesen Bestimmungen, die MNK einer stärkeren Kontrolle, Transparenz und gesellschaftlichen Partizipation unterwerfen wollen, reflektiert das Lima-Statut direkt die ökonomische Situation abhängiger Reproduktion. Fern etwaiger sozialistischer Wirtschaftspolitik unternimmt das Statut eher den Versuch, eine regionale ,,Mexikanisierung“ der Investitionsgesetzgebung zu initiieren ${ }^{90}$. Dabei kann nicht verwundern, daß es eine enorme Ausstrahlungskraft entwickelt hat ${ }^{91}$. Nicht zuletzt sind auch die von privater Seite und den Industrieländern entwickelten Kodizes, die oben diskutiert wurden, auch als Reaktion auf die Entscheidung 24 entstanden. Der reale Effekt des Lima-Statuts ist allerdings bislang gering geblieben. Zurückzuführen ist die geringe Effizienz auf die heterogene politische und ökonomische Ausgangssituation der Mitgliedstaaten. Diese konfligierenden Momente konnten von MNK ausgenutzt werden, um Ausnahmeregelungen durchzusetzen und das Statut zu erodieren. Dieser Prozeß kulminierte in der von der chilenischen Regierung nach dem Militärputsch betriebenen MNK-freundlichen Investitionspolitik, die schließlich zum Austritt Chiles aus dem Andenpakt führte. Die ohnehin schon abnehmende Relevanz des Lima-Statuts verminderte sich noch weiter, da aufgrund der Existenzkrise des Andenpakts, zu der das Ausscheiden Chiles führte, die Entscheidung 24 noch mehr ausgehöhlt wurde ${ }^{92}$. Das Schicksal der Entscheidung 24 zeigt deutlich, daß Absichtserklärungen so lange unzureichend bleiben, wie es gelingt, die beteiligten Staaten politisch und ökonomisch auseinanderzudividieren.

\subsection{Der vietnamesische Investitionskodex von 1977}

In bezug auf die inhaltliche Formulierung kann der vietnamesische Verhaltenskodex ${ }^{93}$ als paradigmatisch für entsprechende Gesetze in Entwicklungsländern angesehen werden ${ }^{94}$. Der Kodex unterscheidet drei Formen der MNK-Tätigkeit in Vietnam: die Kooperation, gemischte Gesellschaften, d. h. Unternehmen, in denen Ausländer 30-49 \% des Kapitals halten, und rein private Unternehmen, die ausschließlich exportorientiert produzieren. Gemischte Gesellschaften haben Rechenschaft abzulegen über Produktionspläne und Entwicklungsperspektiven sowie die Gewinnsituation. Der Kodex gibt MNK eine Eigentumsgarantie für 10-15 Jahre, die ergänzt wird von einem weitgehenden Schutz investierten Kapitals. Danach soll bei eventuellen Enteignungen ein angemessener Preis gezahlt werden, der in einer vernünftigen Frist in der investierten Währung zu entrichten ist. Außerdem wird MNK ein weitgehender Gewinntransfer gestattet. An diesen moderaten Bestimmungen läßt sich der enorme Kapitalbedarf Vietnams ermessen. Den drei obigen Kapitalformen lassen sich unmittelbar ökonomische Funktionen zuordnen, deren Ratio einmal Deckung internen

\footnotetext{
90 Vgl. Oliver, a. a. O. (Anm. 89), S. 784.

91 Vgl. Sauvant, a. a. O. S. 368.

92 Zu Einzelheiten siehe A. Weber, Neuere Tendenzen im Integrationsrecht Lateinamerikas, in: VRU 1978, S. $98 \mathrm{ff}$

93 Reglementation des investissement etrangers en Republique Socialiste du Viet Nam, abgedruckt bei: B. - O. Bryde, Der Kodex über Auslandsinvestitionen der Sozialistischen Republik Vietnam, in: VRU 1978, S. $107 \mathrm{ff}$

94 Vgl. ebd., S. 103. Zu einem Uberblick über derartige Investitionsgesetze siehe E/C. 10 / 8 und Add. 1; Schlupp a. a. O. (Anm. 13), S. $181 \mathrm{ff}$.
} 
Konsumbedarfs und zum anderen Devisenbeschaffung heißt ${ }^{95}$. Während gemischte Gesellschaften in ihrer Produktionspalette perpetuierte interne Konsumtionsmuster berücksichtigen, zielen die beiden anderen Formen auf devisenbeschaffende Produktion ab. So sollen primär mit Erdölkonzernen Kooperationsverträge abgeschlossen werden. Zwar bewerteten die MNK den vietnamesischen Investitionskodex als ,,ermutigend"96, die praktische Resonanz blieb bisher aber doch relativ gering. Gleichwohl ist der Kodex ein notwendiges Mittel, Direktinvestitionen zu erhalten. Problematisch ist es allerdings, wenn sich Vietnam quasi als „Exportsprungbrett“ empfehlen will, da sich für rein private Unternehmen die Kontrollfrage in bezug auf Produktion und Gewinne national kaum lösen läßt. Hinsichtlich der Kontrollmöglichkeiten bildet Vietnam zwar im Vergleich mit anderen Entwicklungsländern kein Paradigma, weil das politische System des Sozialismus viel weitergehende Kontrollmöglichkeiten bietet ${ }^{97}$. Transferpreise z. B. aber lassen sich dennoch national schwerlich kontrollieren. Insofern bilden noch so weitgehende national begrenzte administrative und gesellschaftliche Kontrollen letztlich kein Surrogat für eine internationale MNK-Regulierung.

\section{Realisierungschancen für einen internationalen MNK-Kodex}

Die bis hierhin gemachten Ausführungen zeigen, daß bei allen beteiligten Akteuren grundsätzliche Ubbereinstimmung über die Notwendigkeit eines internationalen Verhaltenskodex für MNK besteht ${ }^{\mathbf{9 8}}$. Erheblicher Dissens besteht allerdings nach wie vor in bezug auf dessen inhaltliche und formale Gestaltung. Im Grunde stehen sich zwei konträre Konzeptionen gegenüber: Wird auf der einen Seite auf Legitimationsbeschaffung $f$ ür MNK gezielt, so zielt die andere Seite auf die Erhöhung der Verhandlungsmacht von Entwicklungsländern. Rein ideell gesehen, unterliegen die Legitimationsinhalte für MNK einem doppelten Wandel. Wurden MNK in der Anfangsphase ihrer Expansion durchweg als positiver Faktor im internationalen Wirtschaftsleben angesehen, so wurden sie mit zunehmender Kapitalakkumulation in steigendem Maße als bedrohlich empfunden ${ }^{99}$. Die angestrebten Verhaltenskodizes nun sollen aus der Sicht der MNK den drohenden Legitimationsschwund stoppen ${ }^{\mathbf{1 0 0}}$. Umgekehrt wird eine Funktionsbestimmung von Verhaltenskodizes aus der Sicht der Entwicklungsländer von der Erhöhung ihrer Verhandlungsmacht gegenüber MNK ausgehen. Die oben referierten Elemente der ILO-Grundsätze, wie sie von der Gruppe der 77 entwickelt wurden, können in diesem Sinn als Minimalforderungen für die MNK-Regulierung verstanden werden.

Inwieweit zwischen diesen beiden Positionen vermittelt werden kann, läßt sich bislang nicht abschätzen. Die Formulierungsbemühungen in der Intergovernmental Working Group jedenfalls verlaufen noch so stockend, daß selbst die definitive Gestaltung des UN-Entwurfs eines Verhaltenskodex noch nicht absehbar ist. Da sich kurzfristig ein einheitlicher, auf der

\footnotetext{
95 Zur ökonomischen Situation und Strategie Vietnams vgl. A. Gordon, Vom Neokolonialismus zum Sozialismus, in: ,,3. Welt Magazin" $1 / 77$, S. $24 \mathrm{ff}$.

$96 \mathrm{Vgl} . \mathrm{NZZ}$ v. 29. 11. 77

97 Davon geht auch die vietnamesische Regierung ganz bewußt aus: Vgl. ,Vietnam - Aufbau des Sozialismus und internationale Politik“, in: ,3. Welt Magazin“ $11-12 / 77$, S. 67.

98 Vgl. auch ,CTC-Reporter“ 4 / 78, S. 6.

99 Diesen Wandel reflektieren direkt und indirekt auch die volkswirtschaftlichen Theorien der Determinanten von Direktinvestitionen; vgl. G. Koopmann, Warum gibt es multinationale Unternehmen?, in: Kebschull/Mayer (Hrsg.). Multinationale Unternehmen, a. a. O. (Anm. 16), S. $36 \mathrm{ff}$.

100 Vgl. zu diesen drei Phasen H. V. Perlmutter, Perplexing Routes to M. N. E. Legitimacy: Codes of Conduct forTechnology Transfer, in: Stanford Journal of International Studies 1976, S. 169 ff. Das hier von Perlmutter entwickelte Phasenmodell wirkt allerdings ausgesprochen trivial, da es grundsätzlich auf jede x-beliebige Problemlösung angewandt werden kann und für den jeweiligen Perzeptionswandel nur verschwommene Erklärungen anbietet.
} 
Basis der eingangs skizzierten maximalen Lösung beruhender Text kaum realisieren läßt, ist eine Funktionsdifferenzierung des Kodex wahrscheinlich, dessen Wahrnehmung bzw. Durchsetzung dann auf verschiedenen Ebenen erfolgt ${ }^{101}$. Weitgehend auf nationaler Ebene würdedanach die Durchsetzung dieses Kodex' erfolgen, die von einer verbesserten regionalen Harmonisierung der Investitionsgesetze flankiert werden sollte. Da auf nationaler Ebene eine effektive Úberwachung der MNK-Tätigkeit letzlich nicht möglich ist, müßte diese Funktion international wahrgenommen werden. Leisten könnte dies das Centre on Transnational Corporations, indem es insbesondere den Entwicklungsländern einen umfassenden Informationsservice anbietet. Wann ein weitergehender Text, also ein universeller, integrierter und mit Bindungswirkung ausgestatteter Kodex, der einem Allgemeinen Investitionsabkommen nahe käme, formuliert werden kann, hängt davon $a b$, inwieweit sich der Verhandlungsspielraum der Entwicklungsländer im internationalen System wieder erweitern läßt.

Obwohl die Realisierungschancen für einen solch weitgehenden Verhaltenskodex momentan gering sind, sollten die positiven Wirkungen, die auch von einem weit unterhalb dieser Schwelle befindlichen Kodex ausgehen, nicht unterschätzt werden. Einmal könnte ein allgemeiner und unverbindlicher internationaler Kodex als blue print fungieren, und durch via Ausstrahlung auf die Gestaltung nationaler Investitionscodes deren Harmonisierung induzieren. Realisieren lassen sich auch relativ weitgehende Rechnungslegungsvorschriften für MNK, was zusätzlich zur Verbesserung des Informationsstandes der Gastländer führen wird. Auf einer eher ideellen Ebene verändert ein solcher Kodex schließlich die gesamte Erwartungshaltung der Gastländer gegenüber MNK. Da insofern durchaus reale, das Verhandlungspotential der Entwicklungsländer durch einen internationalen Kodex erhöhende Möglichkeiten bestehen, ist die Einschätzung, ,,daß den meisten dieser internationalen Aktivitäten (zur Formulierung von Verhaltenskodizes, A. St.) somit eher eine apologetisch-legitimatorische Funktion zukommt" ${ }^{102}$, zu einseitig. Diese Einschätzung, die im Kontext von Senghaas' These steht, die neue Weltwirtschaftsordnung laufe auf einen bloßen ,,symbolic use of politics" hinaus ${ }^{103}$, übersieht wesentliche dynamische Effekte, die in diesen Bestrebungen enthalten sind. Will man nicht in der modellplatonischen Kontemplation der illusionären Gegenstrategie dissoziativer autozentrierter Entwicklung ${ }^{104}$ stehen bleiben, wird man die Veränderung internationaler Wirtschaftsbeziehungen zwischen Industrie- und Entwicklungsländern auf dem Weg von Strukturreformen als sinnvollen Entwicklungsweg anerkennen müssen. Dabei muß sicherlich in Rechnung gestellt werden, daß die Beziehungen zwischen peripherem Staat und MNK nichtantagonistischer Natur sind. Analog zu den Beziehungen des Staates in westlichen Industrieländern zu $\mathrm{MNK}^{\mathbf{1 0 5}}$ ist vielmehr auch in Entwicklungsländern eine tendentiell enger werdende Verflechtung von Staat und MNK zu beobachten. Friktionen treten in diesem Verhältnis aber auf, weil die staatliche Legitimationsbasis national bleibt. Primär dient der (periphere) Staat der Kanalisierung internen Problemdrucks. Während die Entwicklungsländer einerseits auf Industrieländer und MNK zur Entwicklung weitgehend angewiesen sind, müssen sie somit andererseits mit letzteren um eine Umverteilung des international produzierten Mehrprodukts ringen. Elemente, die in diesem partiellen Konflikt die Verhandlungsmacht der Entwicklungsländer vermehren -

101 Vgl. hierzu und zum folgenden: K. P. Sauvant, Controlling ..., (Anm. 39), S. 396 f.

102 Schlupp, a. a. O. (Anm. 13), S. 201. Hiervon nimmt Schlupp nur effektive Maßnahmen zur Informationsverbesserung über MNK aus: ebd., S. $201 / 204$

$103 \mathrm{Vgl}$. ebd., S. 227

104 Vgl. zu dieser Entwicklungsstrategie insbesondere D. Senghaas, Weltwirtschaftsordnung . .., a. a. O. (Anm. 3).

105 Vgl. u. a. A. S. Miller, The Multinational Corporation and the Nation State, in: JW TL 1973, S. 267 ff. 
und hierzu zählen auch Verhaltenskodizes für MNK -, sind folglich für diese Länder geeignete Mittel zum Entwicklungszweck.

Problematisch ist es allerdings, von Verhaltenskodizes eine vollständige und effektive MNK-Kontrolle zu erwarten. Auf der Basis der marktwirtschaftlichen Ordnung können auch umfassende und sehr restriktive Kodizes nur eine relative MNK-Regulierung realisieren. In diesem Zusammenhang erscheint die Forderung nach Wiederherstellung der MNK-Verantwortlichkeit ${ }^{\mathbf{1 0 6}}$ in der Tat zweifelhaft und ,,ideologieverdächtig“. Selbst eine tendentielle Úberwindung des institutionell-organisatorischen Nachholbedarfs peripherer Staaten gegenüber MNK erlaubt keine perfekte Kontrolle dieser Unternehmen. Entscheidend ist vielmehr, daß diese Staaten eine annähernde, wenn auch nur formale Gleichstellung durchsetzen können, die es ihnen ermöglicht, gleiche Ausgangsbedingungen auf dem Weltmarkt herzustellen. Bindende Verhaltenskodizes sind zusammen mit der hierzu komplementären Realisierung ständiger Souveränität über natürliche Ressourcen lediglich die Bedingung sine qua non, um in diesem Sinn die Chancen der Entwicklungsländer im internationalen ,,bargaining process“ gegenüber den anderen Akteuren zu verbessern, und so die Voraussetzung für eine im nationalen Interesse formulierte weitgehend selbstinduzierte Entwicklung zu schaffen ${ }^{\mathbf{1 0 7}}$. Dermaßen funktional verstandene MNK-Kodizes gliedern sich nicht nur bruchlos als eine Facette in die zu schaffende neue Weltwirtschaftsordnung ein, sondern es lassen sich umgekehrt auch Kontrollmaßnahmen für MNK letztlich erst in deren Kontext realisieren. Notwendig ist folglich eine sektoral und territorial umfassende MNKRegulierung, die über Verhaltenskodizes hinausgehend MNK in eine integrierte Kontrollstrategie einbezieht ${ }^{108}$, um MNK auf diesem Weg für eine sinnvolle ökonomische Entwicklung der Dritten Welt nutzbar zu machen.

106 Sauvant, a. a. O. (Anm. 39), S. 359, 399 und passim. Hier besteht im übrigen eine signifikante Parallele zu den verschiedenen Gesetzen gegen Wettbewerbsbeschränkungen. Vgl. R. Knieper, Weltmarkt, Wirtschaftsrecht und Nationalstaat, Frankfurt/M. 1976, Teil drei. 107 Vgl. ,Forum Vereinte Nationen“ April 1978, S. 3

108 Eine derartige integrierte Kontrollstrategie hat Wohlmuth versucht zu entwerfen. Vgl. K. Wohlmuth, Neue Weltwirtschaftsordnung und Transnationale Konzerne. Perspektiven für eine integrierte Kontrollstrategie. in: ders./W. Däubler (Hrsg.), Transnationale Konzerne und Weltwirtschaftsordnung, Baden-Baden 1978, S. $123 \mathrm{ff}$. 


\section{The Question of Ideology in Non-western Societies}

By BASSAM TiBI

In scientific as well as popular literature "ideology" is used synonymously with ideas. In the critique of ideology we can observe normative, subjectivist positions interpreting ideas institutionalized as norms as a determinant of social change. In addition we can observe materialist analyses treating ideologies within the framework of a base-superstructure scheme. The author doubts the usefulness of both approaches for the analysis of ideologies in non-Western societies. He pleads for an approach taking both the immanent structure of ideology and its relation to social evolution into account. In analysing ideologies in nonWestern societies, the researcher is faced with the problem that here ideologies are the product of a process of acculturation and are not simply to be explained from within the context of the autocthonous society. In view of this social reality, the author attempts to develop a different approach to non-western ideologies and concludes with a study of the dominant ideologies of the Middle East: Islam, Arab Nationalism, Arab Socialism, the Arab response to Marxism. In this way the problems of studying ideologies in non-western societies are illustrated. The author shows why the study of these ideologies must be located in two scientific disciplines: the sociology of development and international relations.

\section{The Judiciary and the Bar in India During the Emergency}

By A. G. Noorani

The Janata Party Government's assumption of power initiated a series of post-mortems about the performance of various groups during the emergency. It has been claimed that the legal profession fared the best and the journalists the worst. This appears to be a fairverdict as far as lawyers and High Courts are concerned, while the record of the Supreme Court, due to Indira Ghandi's court packing in 1973 (cf. Sen, Constitutional Storm in India, VRU 1974, p. 33 sq.) has been disappointing. The article discusses in detail the challenges to the emergency legislation by the legal profession, their successes in the High Courts and their frustration in the Supreme Court.

\section{Codes of Conduct for Multinational Corporations in the Light of the Interests of De- veloping Countries}

By AlPHONS STUdieR

The formulation of codes of conduct for multinational corporations is one of the most complex issues that has been discussed within the context of the New International Economic Order. This task covers, among others, the basic issues of balance of payments, technology transfer, employment, transfer prices, taxation and information disclosure which were primarily discussed by the Commission on Transnational Corporations that was created in 1974 under the auspices of the UN-Economic and Social Council. In spite of widespread expectations that such codes sould help to regulate the activities of multinational corporations in developing countries, it is sometimes feared these codes could quite on the contrary prove as ideological means to legitimate these activities. By and large, this objection is true for "weak" codes like the OECD-Guidelines. But on the other hand, codes which are formally binding legal instruments formulated in precise language could really help strengthening the 
"bargaining power" of developing countries vis-à-vis multinational corporations, provided these provisions underline the sovereign rights of host countries. It is necessary, therefore, that these codes stress the principle of permanent sovereignty over natural resources. This article deals with some endeavours to formulate investment codes on an international, regional and national basis in order to analyse their specific scope, language, legal nature and machinery for implementation compared with the aforementioned object. 